\title{
Estrategias de comunicación para enfrentar el ciberacoso en el contexto del COVID-19
}

\author{
Communication strategies for dealing with cyberbullying in the context of \\ COVID-19
}

Miriam Elizabeth Erazo Rodríguez. ${ }^{1}$, Luis Miguel Viñan Carrasco. ${ }^{2}$, Myriam Elizabeth Murillo Naranjo. ${ }^{3}$ \& Cristhy Nataly Jiménez Granizo . ${ }^{4}$

\begin{abstract}
.
DOI: https://doi.org/10.33262/concienciadigital.v4i3.1.1809

Introduction: New technologies and the use of the internet are part of our daily life; they transform language, life and traditional practices. The appearance of COVID-19 has reduced social relations, and the world population faces other types of interaction in all areas. Learning is undoubtedly one of the elements that has had to migrate from face-toface contexts to digital environments, where relationships between users present risky situations, such as digital harassment; There are studies regarding cyberbullying in the educational environment, but the types of strategies used according to the level of the problem are unknown. Objective: Define communication strategies against digital harassment in the context of COVID-19. Methodology: The study is descriptive, qualiquantitative, non-experimental (transactional) design, a literature review was carried out through virtual ethnography to define the accessibility and ease of use of web pages and social networks, to collect information about of the communication strategies, the online

\footnotetext{
${ }^{1}$ Universidad Nacional de Chimborazo, Facultad de Ciencias Políticas y Administrativas, Carrera de Comunicación. Riobamba, Ecuador, miriamerazo@unach.edu.ec, https://orcid.org/0000-0003-15697245

${ }^{2}$ Universidad Nacional de Chimborazo, Facultad de Ciencias Políticas y Administrativas, Carrera de Comunicación. Riobamba, Ecuador, luis.vinan@unach.edu.ec, https://orcid.org/0000-0003-2127-4038

${ }^{3}$ Universidad Nacional de Chimborazo, Facultad de Ciencias Políticas y Administrativas, Carrera de Comunicación. Riobamba, Ecuador, myriammurillo@unach.edu.ec, https://orcid.org/0000-0002-5141$353 \mathrm{X}$

${ }^{4}$ Universidad Nacional de Chimborazo, Facultad de Ciencias de la Educación Humanas y Tecnológicas, Carrera de Pedagogía de la Informática. Riobamba, Ecuador, cjimenez@unach.edu.ec, https://orcid.org/0000-0002-5912-8733
} 
survey was applied, with a reliability of 0.815 , a sample of 247 social communication experts who work in the central area of Ecuador, the analysis of frequencies and Cronbach's Alpha using the SPSS-25 software . Results: The forced interaction, skills for the use of multimedia tools and the alteration of content by the harasser stand out. The communicators' strategies emphasize: Education (audiovisual content), Prevention (monitoring of accounts to avoid impersonation), Evasion (not reacting to the aggressor's comments to reduce the risk), Acceptance (accepting what is requested by the harasser), Confrontation (gathering evidence). Conclusion: The strategies used by social communicators focus on prevention and go as far as confrontation to face cyberbullying according to the level of the problem.

Keywords: cyberbullying; social media; Communication strategies; journalism; covid19

\section{Resumen.}

Introducción: Las nuevas tecnologías y el uso del internet forman parte de nuestra cotidianidad; transforman el lenguaje, la vida y las prácticas tradicionales. La aparición del COVID-19 ha mermado las relaciones sociales, y la población mundial enfrenta otros tipos de interacción en todos los ámbitos. El aprendizaje indudablemente es uno de los elementos que ha tenido que migrar de los contextos presenciales a los entornos digitales, donde las relaciones entre los usuarios presentan situaciones de riesgo, como es el caso del acoso digital; existen estudios respecto al ciberacoso en el entorno educativo, pero se desconocen los tipos de estrategias que se usan de acuerdo con el nivel del problema. Objetivo: Definir estrategias de comunicación frente al acoso digital en el contexto del COVID-19. Metodología: El estudio es descriptivo, cuali-cuantitativo, diseño no experimental (transaccional), se realizó una revisión de la literatura a través de la etnografía virtual para definir la accesibilidad y facilidad del uso de páginas web y redes sociales, para recopilar información acerca de las estrategias comunicativas, se aplicó la encuesta online, con una fiabilidad del 0,815 , muestra de 247 expertos de la comunicación social que laboran en la zona centro del Ecuador, el análisis de las frecuencias y el Alfa de Cronbach mediante el software SPSS-25. Resultados: Resaltan la interacción forzada, habilidades para el uso herramientas multimedia y la alteración de contenidos por parte del acosador. Las estrategias de los comunicadores enfatizan: Educación (contenidos audiovisuales), Prevención (monitorización de las cuentas para evitar la suplantación), Evasión (no reaccionar a los comentarios del agresor para reducir el riesgo), Aceptación (aceptar lo solicitado por el acosador), Enfrentamiento (recopilación de evidencias). Conclusión: Las estrategias que usan los comunicadores sociales se enfocan en la prevención y llegan hasta el enfrentamiento para afrontar el ciberacoso de acuerdo con el nivel del problema.

Palabras claves: ciberacoso; redes sociales; estrategias de comunicación; periodismo; covid-19. 


\section{Introducción.}

La crisis en la que nos vemos abocados por la pandemia del síndrome respiratorio agudo severo por el coronavirus desde el 2019 denominado COVID-19 (Organización Mundial de la Salud, 2020), no solo es un problema sanitaria, sino que ha cambiado el estilo de vida a nivel mundial, provocando la adaptación de las actividades presenciales a lo virtual para evitar el contagio, ahora " las personas dependen más de los dispositivos electrónicos y las redes sociales debido a la implementación de las reglas de distanciamiento social, lo que implica un mayor riesgo de ciberacoso" (Yang, 2021), estamos constantemente expuestos a interacciones digitales que por causa de la pandemia se han ido agudizando, esto nos sitúa evidentemente frente a escenarios inusuales que requieren investigaciones para comprender el fenómeno del ciberacoso en el contexto del COVID-19 y sus los tipos de estrategia que puedan ayudar a las víctimas a enfrentar el problema. El objetivo del presente estudio es definir las estrategias de comunicación para enfrentar el ciberacoso en el contexto del COVID-19 desde la perspectiva de los comunicadores sociales de la zona centro del Ecuador (Riobamba, Ambato, Guaranda).

Ahora bien, las redes sociales son espacios en la red que permiten conectar personas en entornos virtuales, los usuarios comparten información con intereses, fines y usos específicos, en los últimos años su importancia ha sido notable y son objeto de estudio de diferentes disciplinas, "con la llegada de la Web 2.0, las redes sociales en internet ocupan un lugar relevante en el campo de las relaciones personales" (Hernández Guarín \& Castro Pacheco, 2014) sus interacciones están marcadas por varios aspectos particulares como el anonimato total o parcial y lo convierte en un espacio de inseguridad que dan las relaciones que se suscitan por esta vía, como el caso del ciberacoso (Herrera, 2012). Es así que, el avance de las nuevas tecnologías de la información y la comunicación (TIC) y por ende la proliferación de usuarios en las redes, es común ver interacciones sociales que involucran contenido ofensivo en línea, ya que es una de las principales expresiones de agresión en situaciones de hostigamiento cibernético, conocido en la actualidad como acoso cibernético (Rosa et al., 2019).

Durante la pandemia de COVID-19, la interconexión digital sin precedentes y la intensa cobertura de los medios han amplificado el miedo psicológico asociado a la pandemia (Chiolero, 2020), las medidas adoptadas por los países implican entre otras alternativas el uso de medios de comunicación digital, donde se consolidan espacios de exposición a nuevos riesgos. Los usuarios se infiltran en la vida de las personas, aprovechan el poder que tienen para realizar actividades no consentidas con otras, a través de plataformas tecnológicas de comunicación, se presenta nuevas formas de relación social online (Torres, Manuel, \& de Marco, 2013) que afectan y cambian la vida de las personas: en sus comportamientos, actividades cotidianas que como individuos sociales les caracteriza $y$, por otro lado, vemos como estas relaciones sociales estructuralmente han cambiado sus actividades hacia un contexto digital de internet y las redes sociales. Es así como el problema del acoso, "es un comportamiento agresivo y no deseado entre niños/as en edad escolar que involucra un desequilibrio de poder real o percibido" (Verdejo, 2015) mientras que el ciberacoso es considerado como: 
Un conjunto de comportamientos mediante los cuales una persona, un conjunto de ellas o una organización usan las TIC para hostigar a una o más personas. Dichos comportamientos incluyen, aunque no de forma excluyente, amenazas y falsas acusaciones, suplantación de la identidad, usurpación de datos personales, daños al ordenador de la víctima, vigilancia de las actividades de la víctima, uso de información privada para chantajear a la víctima, etc. (Bocij, Boci, \& Mcfarlane, 2002).

De hecho, tanto el acoso como el ciberacoso tienen una relación que involucra situaciones emocionales asociadas a problemas psicológicos. El ciberacoso es una problemática de la sociedad digital que vulnera la intimidad (Tello, 2013) y se caracteriza por la comunicación intensa y maliciosa; podría integrar presiones, expresiones discriminatorias (Dueñas, Ponton, Belzunegui, \& Pastor, 2016) así como también: intimidación, ofensas, extorsión y más características maliciosas, mediante comentarios o mensajes (Cañón, Grande, \& Ferrero, 2018), además el uso de material multimedia desarrollado y difundido por uno o varios usuarios en las redes sociales para infundir temor o perjudicar a una $o$ varias víctimas.

El acoso en internet requiere de conocimientos avanzados de la tecnología, abuso de poder, agresiones constantes (González, 2016) que pueden provocar ansiedad social (Ruíz-Martín, Bono-Cabré, \& Magallón-Neri, 2019), provocando incluso el suicidio de la víctima (Young, Subramanian, Miles, Hinnant, \& Andsager, 2017). La violencia digital presente en la sociedad de la información se instaura en la vida cotidiana, el acoso offline y online como lo hemos mencionado puede generar graves consecuencias en la estabilidad psicológica de las víctimas, esto porque los usuarios están supeditados a la irrupción sistemática y con conjeturas de odio de usuarios no reconocidos que provocan miedo por disímiles medios con el fin de lograr sus objetivos. (Obermaier, Hofbauer, \& Reinemann, 2018).

Es evidente que la violencia en las redes sociales está presente, los usuarios que interaccionan en estos contextos son individuos con formación heterogénea y muchos de ellos utilizan el anonimato para expresarse y son confundidas con la libertad de expresión, "los discursos que se manejan en estos espacios carecen de un mínimo de racionalidad y respeto en el lenguaje y las formas de comunicarse" (Carrera \& Morejón, 2019), por lo tanto, cuando alguien se comunica de forma violenta, lo que acontece es que se pone en riesgo y se anula la capacidad de continuidad que tiene la comunicación. La violencia rompe la comunicación, entendida en su sentido más propositivo.

El uso de herramientas digitales de prevención e intervención en el contexto del ciberacoso, debe instaurarse como una práctica común para evitar que las personas reciban contenido en línea dañino en las redes sociales (Rosa et al., 2019), particularmente entre los adolescentes y grupos más vulnerables. Si bien estas herramientas no son la solución a este problema social, pueden ayudar a reducir la incidencia del ciberacoso en múltiples contextos. De ahí, la importancia de integrar las herramientas digitales a través del uso de estrategias comunicacionales en aplicaciones, juegos, sitios web, redes sociales 
entre otras, para prevenir el ciberacoso o detectarlo a tempranamente, evitando así comportamientos dañinos. Esta integración de herramientas digitales con estrategias comunicaciones se puede complementar además con interfaces reflectantes, que promuevan la reflexión de los usuarios e impulse un adecuado comportamiento en línea, así como interacciones positivas.

El problema del ciberacoso ha sido evidenciado en múltiples investigaciones (Dinakar, Reichart, \& Lieberman, 2011) (Van Cleemput, K., Vandebosch, Poels, K., Bastiaensens, DeSmet, \& De Bourdeaudhuij, 2015) (Van Royen, Poels, Vandebosch, \& Adam, 2017) estableciendo además la necesidad de contar con herramientas digitales capaces de identificar correctamente el ciberacoso, distinguiéndolo de otras situaciones en línea como el uso de lenguaje soez o palabras malsonantes entre compañeros en un contexto lúdico.

Dicho esto, y bajo la consideración que la comunicación actualmente ha alcanzado mucha fuerza a través del entorno digital (Carrera \& Morejón, 2019), resulta necesario proponer estrategias para enfrentar el problema y conocer la opinión de profesionales de la comunicación con respecto a las ventajas y desafíos de las nuevas Tecnologías de la Información y Comunicación.

De acuerdo con Bégin (2018), para lograr el desarrollo de estrategias efectivas de cambio social en atención al problema de ciberacoso, es importante abordarlo desde un enfoque comunicacional y temporal, que tome en cuenta las opiniones de los actores involucrados en estos escenarios. El fenómeno comunicacional de ciberacoso no representa situaciones unidireccionales, sino que también se presenta como un tema de poder entre los usuarios, ocasionando problemas entre ellos, cuyo proceso es proclive a modificaciones en función del tiempo y los contextos de interacción.

Por otra parte, el avance de estrategias de comunicación frente al ciberacoso involucra otros aspectos y orientaciones para su desarrollo:

- La investigación no solo debe centrarse en la relación agresor y víctima (González, 2016), también se debe tomar en cuenta el efecto de los observadores que contemplan las agresiones sin hacer nada al respecto (Domínguez, MartínezPecino, \& Segura, 2015).

- Las víctimas deben conocer las leyes y normativas vigentes que las amparan para defenderse (Cerezo \& Rubio, 2017).

- Es importante considerar el alto consumo de contenido audiovisual en las redes sociales (García, Tur-Viñes, \& Pastor, 2018), es un efectivo recurso didáctico (Ballesteros, 2017) que permitirá enseñar acerca de los riesgos y prevención (Vanderhoven, Schellens, \& Valcke, 2014).

- Se debería considerar a los grupos de apoyo online como estrategias de intervención contra el ciberacoso en redes sociales (Tudela \& Barón, 2017). 
- Es necesario que los usuarios tengan competencias digitales para afrontar los retos del ciberacoso y reducir la brecha digital (Peral-Peral, Arenas-Gaitán, \& VillarejoRamos, 2015).

Finalmente, es importante reflexionar acerca del papel que tiene la educación, en el uso y empleo de las TIC como herramientas que posibilitan el desarrollo de capacidades cognitivas, en los ámbitos sociales, políticos y económicos, que además pauten y establezcan los protocolos necesarios para un uso seguro de internet y permitan aprovechar sus beneficios y minimizar sus riesgos. Dentro de este contexto es importante entender, que el uso de dispositivos tecnológicos se ha convertido en una práctica habitual y cotidiana, que transforman las relaciones entre pares, es por eso que su aplicación puede ser ventajosa o negativa según se use, por eso lo tanto se debe manejar adecuadamente las tecnologías para aprovechar sus beneficios y disminuir sus riesgos (Verdejo, 2015).

\section{Metodología.}

La investigación se basa en el enfoque cuantitativo (Hernández-Sampieri, FernándezCollado, \& Baptista-Lucio, 2010), de tipo descriptivo, con un diseño no experimental transaccional. La principal técnica utilizada es la encuesta online para la obtención de datos en el período de tiempo establecido entre el 28 de septiembre y 6 de noviembre del 2020. El instrumento utilizado consistió en un cuestionario con una fiabilidad del 0,815 y cuyas preguntas presentan opciones de respuesta con escala de tipo Likert (1: Total desacuerdo / 2: En desacuerdo / 3: Indiferente / 4: De acuerdo / 5: Total acuerdo).

A través de un muestreo por conglomerado aleatorio, se evaluaron 29 indicadores agrupados en 8 secciones: (1) Caracterización del ciberacoso, (2) herramientas multimedia, (3) educación, (4) estrategia de prevención, (5) estrategia de evasión, (6) estrategia de aceptación, (7) estrategia de enfrentamiento y, (8) análisis de fiabilidad. Los datos se analizaron con el software SPSS-25 (frecuencias y Alfa de Cronbach).

En el estudio participaron 687 comunicadores sociales con experiencia en el tema de estudio y que laboran en medios e instituciones públicas y privadas de la zona donde se realizó la investigación, el 62,35\% corresponde a hombres y el 37,65\% a mujeres.

Fases del estudio:

Primera fase:

- Inicia con el análisis del problema del ciberacoso en las redes sociales (netnografía) y la detección de necesidades que ayuden a frenar el problema.

- Revisión bibliográfica (Kitchenham, 2004) para comprender el estado actual de las investigaciones, que se contrastará con la realidad, para identificar las necesidades y luego realizar la entrevista a expertos.

- Entrevistas a profundidad a los profesionales de comunicación para analizar las estrategias, variables e indicadores de estudio y contrastar con la literatura científica. 
- Definición de las variables e indicadores del estudio con el panel de expertos en varias rondas de análisis.

Segunda fase:

- Panel de expertos con los comunicadores

- Diseñó del cuestionario y validación del instrumento

- Aplicación de encuesta online a la muestra (comunicadores de Riobamba, Ambato, Bolívar) para cuantificar las respuestas e identificar las frecuencias.

- Análisis de frecuencias de los datos y el Alfa de Cronbach para medir la fiabilidad del instrumento con el software SPSS versión 25.

- Redacción del informe de investigación y publicación

\section{Resultados.}

Los resultados se han dividido en tres secciones: Sección ciberacoso: a) Caracterización y b) Herramientas multimedia. Sección estrategias: c) Educativa, d) prevención, e) Evasión, f) Aceptación y g) Enfrentamiento. Sección Fiabilidad: h) Estadísticas.

Caracterización del ciberacoso: En las frecuencias (Tabla 1) se observa que las características del ciberacosador, identificadas en la escala con total acuerdo y de acuerdo por los comunicadores. El problema: (1) los actos de violencia digital que tienen como base las patologías psicológicas que de los ciberacosadores y que le impulsan a cometer delitos el entorno digital, (2) los ciberacosadores se aprovechan de la información digital en internet y los contactos disponibles en las redes sociales para encontrar a sus víctimas, (3) cuando las victimas están siendo acosadas en las redes sociales, los usuarios suelen observar, pero no ayudan a frenar el acoso digital, (5) los acosadores forzan a las victima a una mayor interacción basados en la extorsión o intimidación, (6) el ciberacoso es muy frecuente en las redes sociales, esta es una problemática cultural que tiene que ver con el morbo que tienen los usuarios en el desarrollo de conflictos y en algunos casos apoyan al acosador, (7) existe un desconocimiento en normas de buen comportamiento digital (netiqueta) y educación por parte de los usuarios de las redes sociales, (8) los usuarios de internet se sienten desprotegidos en las redes sociales por las limitadas sanciones legales y en algunas plataforma ninguna herramienta para denunciar el ciberacoso amparadas en el marco legal para sancionar a los ciberacosadores.

En total desacuerdo y en desacuerdo encontramos el problema del ítem (4) en el que para continuar con la violencia digital los ciberacosadores manipulan a los usuarios de las redes sociales a compartir publicaciones y continuar con las agresiones multimedia.

Tabla 1. Caracterización del ciberacoso

\begin{tabular}{clccccc}
\hline $\mathbf{N}$. & Ítems & $\mathbf{1}$ & $\mathbf{2}$ & $\mathbf{3}$ & $\mathbf{4}$ & $\mathbf{5}$ \\
\hline 1 & $\begin{array}{l}\text { Problemas } \\
\text { psicológicos }\end{array}$ & $24,29 \%$ & $10,12 \%$ & $5,26 \%$ & $10,93 \%$ & $\mathbf{4 9 , 3 9 \%}$ \\
2 & $\begin{array}{l}\text { Identificar } \\
\text { victimas }\end{array}$ & $13,77 \%$ & $12,96 \%$ & $4,86 \%$ & $10,93 \%$ & $\mathbf{5 7 , 4 9 \%}$ \\
\hline
\end{tabular}




\begin{tabular}{lllllll}
\hline 3 & $\begin{array}{l}\text { Comunidad } \\
\text { indiferente }\end{array}$ & $13,77 \%$ & $12,15 \%$ & $13,77 \%$ & $\mathbf{3 6 , 8 4 \%}$ & $23,49 \%$ \\
4 & $\begin{array}{l}\text { Manipular a } \\
\text { la } \\
\text { comunidad }\end{array}$ & $\mathbf{2 8 , 7 4 \%}$ & $10,93 \%$ & $12,55 \%$ & $23,48 \%$ & $\mathbf{2 4 , 2 9 \%}$ \\
5 & $\begin{array}{l}\text { Interacción } \\
\text { forzada }\end{array}$ & $6,07 \%$ & $10,53 \%$ & $6,48 \%$ & $14,57 \%$ & $\mathbf{6 2 , 3 5 \%}$ \\
6 & Cultura & $17,00 \%$ & $11,34 \%$ & $12,15 \%$ & $\mathbf{3 8 , 0 6 \%}$ & $\mathbf{2 1 , 4 6 \%}$ \\
7 & Netiqueta & $13,77 \%$ & $15,79 \%$ & $10,53 \%$ & $\mathbf{2 8 , 3 4 \%}$ & $\mathbf{3 1 , 5 8 \%}$ \\
8 & Falta de & $7,29 \%$ & $10,12 \%$ & $12,55 \%$ & $\mathbf{4 0 , 8 9 \%}$ & $\mathbf{2 9 , 1 5 \%}$ \\
\hline
\end{tabular}

Fuente: Elaboración propia

Herramientas de acoso digital: En las herramientas de acoso digital, se observa un total acuerdo y de acuerdo (Tabla 2) respecto a que (9) los acosadores con mala intensión comentan, colocan reacciones, stickers, fotos y etiquetas en las publicaciones de las víctimas, (10) también envían intensamente mensajes con contenido persuasivo multimedia (textos, fotos, videos, otros) a sus víctimas, (12) para suplantar la identidad digital y enviar publicaciones maliciosas a los contactos de sus víctimas; hurtan las cuentas en las en redes sociales, (13) también editan con intenciones perversas el contenido publicado por las víctimas y comparten por redes sociales. No es muy frecuente y presenta un total desacuerdo que (11) en las historias publicadas por las víctimas, los acosadores responden para ejercer presión, molestar o hacerles daño.

Tabla 2. Herramientas de acoso digital

\begin{tabular}{|c|c|c|c|c|c|c|}
\hline N. & Ítems & $\mathbf{1}$ & 2 & 3 & 4 & 5 \\
\hline 9 & $\begin{array}{l}\text { Comentarios } \\
\text { en } \\
\text { publicaciones }\end{array}$ & $19,43 \%$ & $10,53 \%$ & $12,15 \%$ & $31,17 \%$ & $26,72 \%$ \\
\hline 10 & $\begin{array}{l}\text { Mensajes de } \\
\text { chat }\end{array}$ & $8,50 \%$ & $14,98 \%$ & $14,57 \%$ & $16,19 \%$ & $45,75 \%$ \\
\hline 11 & Historias & $28,74 \%$ & $13,36 \%$ & $14,57 \%$ & $23,89 \%$ & $19,43 \%$ \\
\hline 12 & $\begin{array}{l}\text { Robo de } \\
\text { cuentas }\end{array}$ & $10,12 \%$ & $8,50 \%$ & $12,96 \%$ & $42,91 \%$ & $25,51 \%$ \\
\hline 13 & $\begin{array}{l}\text { Alteración del } \\
\text { contenido }\end{array}$ & $7,69 \%$ & $10,53 \%$ & $5,26 \%$ & $17,41 \%$ & $59,11 \%$ \\
\hline
\end{tabular}

Fuente: Elaboración propia

Alfabetización digital: En relación con la alfabetización digital (Tabla 3) se evidencia un total acuerdo y de acuerdo con la necesidad de (14) prevención del ciberacoso en redes sociales, mediante la producción de contenidos audiovisuales que contribuyan a la sensibilización de los usuarios en las redes sociales mediante la exposición del método que usan los ciberacosadores y casos de la vida real, (15) capacitar en técnica de comunicación asertiva, resiliente y protección legal, para que los usuarios comprendan la forma de comunicarse cuando enfrentan problemas de ciberacoso y no se pongan en riesgo, (16) también es necesario capacitar a los usuarios de internet para el desarrollo de competencias digitales y ayudar a proteger sus dispositivos electrónicos, el software o 
aplicaciones que usan a diario y su información digital multimedia que publican en internet para frenar el acceso a la información de podría tener los ciberacosadores.

Tabla 3. Alfabetización digital

\begin{tabular}{|c|c|c|c|c|c|c|}
\hline N. & Ítems & $\mathbf{1}$ & 2 & 3 & 4 & 5 \\
\hline 14 & $\begin{array}{l}\text { Contenido } \\
\text { audiovisual }\end{array}$ & $2,83 \%$ & $6,07 \%$ & $7,69 \%$ & $10,93 \%$ & $72,47 \%$ \\
\hline 15 & $\begin{array}{l}\text { Comunicación } \\
\text { asertiva }\end{array}$ & $10,93 \%$ & $8,50 \%$ & $11,34 \%$ & $11,74 \%$ & $57,49 \%$ \\
\hline 16 & Informática & $11,74 \%$ & $16,60 \%$ & $14,57 \%$ & $36,03 \%$ & $21,05 \%$ \\
\hline
\end{tabular}

Fuente: Elaboración propia

Estrategia de prevención: Existe un total acuerdo y de acuerdo (Tabla 4) con la necesidad de (17) reducir al máximo la comunicación por mensajes y actividades online con los ciberacosadores ya que intentan constantemente llamar la atención y saber si tiene la atención de sus víctimas, (19) también se debe revisar las notificaciones, inicio de sesión, publicaciones y todas las actividades realizadas en sus cuentas de redes sociales para evitar la suplantación de identidad y mantener la seguridad, (20) verificar la veracidad de las cuentas de usuarios que envían solicitudes de amistad en las redes sociales y en lo posible no aceptar a personas desconocidas, (21) muchas veces envían documentos adjuntos en los mensajes o hipervínculos, pero es necesario tomar medidas de precaución, verificar el formato de archivo o dirección web a la que se va a acceder y en lo posible no ejecutar el documento o dar permisos de administración ya que puede ser un virus informático y generar serios problemas. Existe indiferencia respecto a que (18) no todas las publicaciones en redes sociales deben ser para todo el público, es mejor limitar el alcance y mucha información personal (biografía, información personal, amigos, fotos, ubicación y etiquetado) debe ser activada la privacidad.

Tabla 4. Estrategia de prevención

\begin{tabular}{|c|c|c|c|c|c|c|}
\hline N. & Ítems & $\mathbf{1}$ & 2 & 3 & 4 & 5 \\
\hline 17 & Provocaciones & $7,29 \%$ & $9,72 \%$ & $10,93 \%$ & $11,74 \%$ & $60,32 \%$ \\
\hline 18 & Privacidad & $10,12 \%$ & $13,36 \%$ & $27,53 \%$ & $25,10 \%$ & $23,89 \%$ \\
\hline 19 & Suplantación & $9,72 \%$ & $12,15 \%$ & $8,50 \%$ & $6,88 \%$ & $62,75 \%$ \\
\hline 20 & Desconocidas & $14,57 \%$ & $19,84 \%$ & $23,08 \%$ & $25,10 \%$ & $17,41 \%$ \\
\hline 21 & Virus & $21,05 \%$ & $13,77 \%$ & $20,24 \%$ & $17,81 \%$ & $27,13 \%$ \\
\hline
\end{tabular}

Fuente: Elaboración propia

Evasión: En la (Tabla 5) se muestra un total acuerdo y de acuerdo en relación con que (24) no reaccionar a los comentarios de los acosadores para minimizar los riesgos e intentos de provocación, (25) prefiero eliminar mis cuentas, aunque tenga que perder mis seguidores y publicaciones para limitar la comunicación por redes sociales con los ciberacosadores. La mayoría está en desacuerdo y total desacuerdo (22) por la presión y el recelo a la venganza de los acosadores virtuales y no se denuncian, (23) no respondo a 
los comentarios mensajes que me envían los ciberacosadores pero si reviso y analizo que dicen y que intentan hacer.

Tabla 5. Estrategia de evasión

\begin{tabular}{clccccc}
\hline $\mathbf{N}$ & Ítems & $\mathbf{1}$ & $\mathbf{2}$ & $\mathbf{3}$ & $\mathbf{4}$ & $\mathbf{5}$ \\
\hline $\mathbf{2 2}$ & Miedo & $\mathbf{1 1 . 7 4 \%}$ & $\mathbf{2 4 , 7 0 \%}$ & $22,67 \%$ & $20,24 \%$ & $20,65 \%$ \\
23 & Revisar & $\mathbf{3 4 , 8 2 \%}$ & $25,10 \%$ & $15,38 \%$ & $7,69 \%$ & $17,00 \%$ \\
24 & Evitar & $12,55 \%$ & $7,29 \%$ & $4,45 \%$ & $7,69 \%$ & $\mathbf{6 8 , 0 2 \%}$ \\
25 & Cerrar & $22,27 \%$ & $13,77 \%$ & $21,46 \%$ & $16,19 \%$ & $\mathbf{2 6 , 3 2 \%}$ \\
\hline
\end{tabular}

Fuente: Elaboración propia

Aceptación: En la (Tabla 6) se evidencia la respuesta en ocasiones y presenta un total acuerdo (26) estoy pendiente y reviso cada mensaje que me envía el ciberacosador para responder, (28) para que no se moleste el ciberacosador he aceptado participar en las actividades virtuales que me pide, tales como chats, foros, videoconferencias, etc. Se observa indiferencia (27) al ciberacosador le he compartido contenido digital que pide (fotos, gráficos, textos, grabaciones de video, audio, etc.).

Tabla 6. Estrategia de aceptación

\begin{tabular}{clccccc}
\hline N. & Ítems & $\mathbf{1}$ & $\mathbf{2}$ & $\mathbf{3}$ & $\mathbf{4}$ & $\mathbf{5}$ \\
\hline 26 & Responder & $10,53 \%$ & $12,96 \%$ & $13,77 \%$ & $22,67 \%$ & $\mathbf{4 0 , 0 8 \%}$ \\
27 & Compartir & $18,22 \%$ & $19,03 \%$ & $\mathbf{2 2 , 6 7 \%}$ & $19,03 \%$ & $21,05 \%$ \\
28 & Actividades & $16.19 \%$ & $9.31 \%$ & $4.45 \%$ & $21.05 \%$ & $\mathbf{4 8 . 9 9 \%}$ \\
\hline
\end{tabular}

Fuente: Elaboración propia

3.7 Enfrentamiento: En la Tabla 7 vemos un alto porcentaje de aceptación para la estrategia de enfrentamiento (ítem 29, 30, 31, 32 y 33), el análisis de los intereses del acosador para saber cómo defenderse, el otro es el apoyo la comunidad virtual, la recopilación de evidencias multimedia del ciberacoso para usar en el proceso judicial, realizar la denuncia formal del ciberacoso ante las autoridades para que se procese la investigación y sanción del acosador, el bloqueo del contacto del acosador en la red social y reportar el problema a los administradores de la red social para que cierren la cuenta.

Tabla 7. Estrategia de enfrentamiento

\begin{tabular}{llccccc}
\hline $\mathbf{N}$. & Ítems & $\mathbf{1}$ & $\mathbf{2}$ & $\mathbf{3}$ & $\mathbf{4}$ & $\mathbf{5}$ \\
\hline 29 & Interés & $9,31 \%$ & $17,00 \%$ & $10,93 \%$ & $\mathbf{3 7 , 6 5 \%}$ & $\mathbf{2 5 , 1 0 \%}$ \\
30 & Apoyo & $8.91 \%$ & $9.31 \%$ & $11.34 \%$ & $\mathbf{3 6 . 8 4 \%}$ & $33.60 \%$ \\
31 & Evidencias & $24,29 \%$ & $14,98 \%$ & $14,17 \%$ & $13,36 \%$ & $\mathbf{3 3 , 2 0 \%}$ \\
32 & Denuncia & $7.29 \%$ & $15.79 \%$ & $12.15 \%$ & $\mathbf{4 1 . 3 0 \%}$ & $23,08 \%$ \\
33 & Bloquear & $15,38 \%$ & $17,41 \%$ & $17,00 \%$ & $\mathbf{2 2 , 2 7 \%}$ & $\mathbf{2 7 , 9 4 \%}$ \\
\hline
\end{tabular}

Fuente: Elaboración propia

Fiabilidad del test: En el estadístico del total de elementos (Tabla 8) se evidencia una alta correlación del total de elementos con el Alfa de Cronbach, hay que aclarar que si algún elemento se suprime se podría elevar su valor (ítem 11, 14, 16, 27, 32, 33), pero al 
ser elementos importantes para la definición de las estrategias, debido a que no hay afectación entonces se mantiene los ítems.

Tabla 8. Estadísticas del total de elemento

\begin{tabular}{|c|c|c|c|c|}
\hline & $\begin{array}{c}\text { Media de } \\
\text { escala si el } \\
\text { elemento se } \\
\text { ha } \\
\text { suprimido } \\
\end{array}$ & $\begin{array}{c}\text { Varianza de } \\
\text { escala si el } \\
\text { elemento se } \\
\text { ha } \\
\text { suprimido } \\
\end{array}$ & $\begin{array}{l}\text { Correlación } \\
\text { total de } \\
\text { elementos } \\
\text { corregida }\end{array}$ & $\begin{array}{c}\text { Alfa } \\
\text { de Cronbach si } \\
\text { el elemento se } \\
\text { ha suprimido }\end{array}$ \\
\hline $\begin{array}{l}\text { 1. Los problemas } \\
\text { psicológicos } \\
\text { del ciberacosador le llevan } \\
\text { a cometer actos de } \\
\text { violencia digital (ira, } \\
\text { venganza, etc.) y delitos }\end{array}$ & 112,85 & 284,854 & 0,292 & 0,811 \\
\hline $\begin{array}{l}\text { 2. El ciberacosador busca } \\
\text { contenido en la red para } \\
\text { identificar a las víctimas } \\
\text { 3. Las comunidades } \\
\text { virtuales son observadores, } \\
\text { indiferentes ante } \\
\text { el ciberacos y no defienden } \\
\text { a las víctimas }\end{array}$ & 112,91 & 286,731 & 0,297 & 0,814 \\
\hline $\begin{array}{l}4 . \\
\text { El ciberacosador manipula } \\
\text { a la comunidad virtual para } \\
\text { compartir contenido, } \\
\text { comentar, asociarse a su } \\
\text { causa y seguir atacando a } \\
\text { la víctima }\end{array}$ & 113,32 & 288,552 & 0,254 & 0,812 \\
\hline $\begin{array}{l}\text { El cibeacosador incrementa } \\
\text { las interacciones forzada } \\
\text { contra la víctima para } \\
\text { intimidar }\end{array}$ & 112,19 & 280,301 & 0,529 & 0,803 \\
\hline $\begin{array}{l}\text { 6. La cultura del acoso es } \\
\text { común en las redes } \\
\text { sociales y entretiene a los } \\
\text { miembros de la comunidad } \\
\text { virtual }\end{array}$ & 113,13 & 308,614 & $-0,125$ & 0,824 \\
\hline $\begin{array}{l}\text { 7. Falta de educación en } \\
\text { netiqueta para un mejor } \\
\text { comportamiento de los } \\
\text { miembros de las } \\
\text { comunidades virtuales en } \\
\text { las redes sociales }\end{array}$ & 113 & 282,089 & 0,443 & 0,805 \\
\hline $\begin{array}{l}\text { 8. Falta de leyes y } \\
\text { herramientas para } \\
\text { denunciar } \\
\text { el ciberacoso en las redes } \\
\text { sociales y que protejan a } \\
\text { los usuario }\end{array}$ & 112,61 & 285,905 & 0,429 & 0,807 \\
\hline $\begin{array}{l}\text { 9. Los acosadores } \\
\text { comentan maliciosamente } \\
\text { las publicaciones de las } \\
\text { victimas mediante } \\
\text { reacciones, stickers, fotos y } \\
\text { etiquetas }\end{array}$ & 113 & 287,126 & 0,308 & 0,81 \\
\hline $\begin{array}{l}\text { 10. Los acosadores envían } \\
\text { intensamente mensajes } \\
\text { con contenido persuasivo } \\
\text { multimedia (textos, fotos, } \\
\text { videos, otros) a sus } \\
\text { víctimas }\end{array}$ & 112,59 & 274,178 & 0,616 & 0,799 \\
\hline
\end{tabular}


11. Los acosadores responden en las historias de sus víctimas para presionar, molestar o hacerle daño de forma constante

12. Los acosadores roban las cuentas de sus víctimas para suplantar la identidad digital y realizar

publicaciones maliciosas a los contactos de las redes sociales

13. Los acosadores alteran maliciosamente el contenido multimedia de la víctima para difundir con los contactos de su redes sociales

14. Crear contenido audiovisual educativo que permita sensibilizar a las comunidades virtuales mediante casos reales y prevenir el ciberacoso en redes sociale

15. Capacitar en técnica de comunicación

asertiva, resiliencia y leyes para la protección, que ayuden a reducir el riesgo en los comentarios con el ciberacosador

16. Capacitación informática para la protección de dispositivos, aplicaciones y protección del contenido multimedia contra el ciberacoso

17. Se debe reducir al máximo la interacción con el ciberacosador, no responder a los mensajes o provocaciones, ya que trata de provocar y saber si tiene la atención de la víctima 18. Revisar su información publicada y configurar la privacidad del contenido de su biografía, información personal, amigos, fotos, ubicación y etiquetado en sus redes sociales

19. Monitorear constantemente la seguridad, inicio de sesión y notificaciones de su cuenta para evitar suplantación de identidad

20. No aceptar la solicitud de amistad de personas desconocidas de su grupo de amigos reales

21. No abrir documentos o hipervínculos que le envíen por mensajes ya que puede ser un virus informático

22. No solicita ayuda a las autoridades por miedo a las represalias

del ciberacosado
113,44

296,076

0,119

0,817

112,7

290,437

0,301

0,81

281,876

0,471

0,805

$\begin{array}{llll}111,9 & 300,34 & 0,084 & 0,816\end{array}$

$\begin{array}{llll}112,39 & 277,76 & 0,524 & 0,802\end{array}$

$\begin{array}{llll}112,97 & 303,028 & -0,003 & 0,82\end{array}$

$\begin{array}{llll}112,28 & 279,412 & 0,528 & 0,803\end{array}$

$\begin{array}{llll}112,96 & 288,149 & 0,346 & 0,809\end{array}$

112,35

277,488

0,521

0,802

113,25

290,959

0,266

0,812

113,19

293,336

0,177

0,815

113,22

292,19

0,237 
ISSN: 2600-5859

23. Reviso todos los mensajes

del ciberacosador pero no respondo

24. Evito reaccionar a los comentarios

del ciberacosador para

reducir el riesgo

25. He cerrado las cuentas de mis redes sociales para cortar la comunicación con los ciberacosadores, pero he perdido la red de contactos

26. Respondo a todos los mensajes del acosador

27.Comparto información multimedia que solicita el ciberacosador (fotos, gráficos, textos, grabaciones de video, audio, etc.)

28. Realizar actividades online que solicita el ciberacosador para que no se moleste (participar en chats, foros, videoconferencias, etc.)

29. Analizar lo que le interesa

al ciberacosador para saber cómo defenderse

30. Solicito apoyo a los amigos, conocidos y otros usuarios de la comunidad virtual, ya sea mediante mensajes privados 0 mostrando evidencias en varias publicaciones para recibir el apoyo que necesito

31. Recopilo evidencias multimedia

del ciberacoso para usar en el proceso judicial

32. Con las evidencias multimedia, realizar la denuncia formal

del ciberacoso ante las autoridades para que se procesa en la investigación y sanción

del ciberacosador

33. Bloqueo el contacto del ciberacosador en mi red social y reporto el problema a los administradores de la red social para que cierren la cuenta

\begin{tabular}{|c|c|c|c|}
\hline 113,89 & 291,272 & 0,225 & 0,813 \\
\hline 112,24 & 280,128 & 0,456 & 0,805 \\
\hline 113,25 & 289,335 & 0,256 & 0,812 \\
\hline 112,67 & 273,816 & 0,63 & 0,798 \\
\hline 113,3 & 296,479 & 0,128 & 0,816 \\
\hline 112,58 & 280,724 & 0,422 & 0,806 \\
\hline 112,83 & 281,643 & 0,493 & 0,804 \\
\hline 112,59 & 286,666 & 0,384 & 0,808 \\
\hline 113,19 & 286,637 & 0,284 & 0,811 \\
\hline 112,77 & 300,721 & 0,056 & 0,818 \\
\hline 113,06 & 295,883 & 0,135 & 0,816 \\
\hline
\end{tabular}

Fuente: Elaboración propia

\section{Conclusiones.}

El análisis realizado en esta investigación, de acuerdo con los resultados presentados en las 5 secciones: Principios del ciberacoso, Educación preventiva, Privacidad preventiva, 
Herramientas multimedia, Gestión del ciberacoso, desde la perspectiva de la comunicación, muestra que el ciberacoso está:

- Caracterizado por los problemas psicológicos del acosador, la identificación de las víctimas, la indiferencia de la comunidad virtual, las interacciones forzadas, la falta de educación en netiqueta y la inexistencia de leyes para la protección de los usuarios, y es que algunas personas han encontrado en la tecnología una vía idónea y certera para ejercer nuevas formas de delinquir, esto es por los vacíos legislativos que existen, a los cambios de las leyes y la falta de conocimiento acerca del peligro que puede ocasionar su uso.

- En relación con las herramientas multimedia de ciberacoso más usadas tenemos a los comentarios en publicaciones, los mensajes de chat, el robo de cuentas y el compartir publicaciones, son indudables y evidentes los peligros que traen consigo el uso de dispositivos móviles, por lo que es necesaria la orientación respecto al uso de estos dispositivos, y deben implementarse iniciativas para su manejo apropiado.

- En la estrategia educativa se observa mayor relevancia en contenido, comunicación y la informática, por esto es importante que las instituciones educativas cuenten con programas de capacitación y los indicios y realizar las denuncias.

- La estrategia de prevención se centra en la reducción de la interacción con el acosador para evitar las provocaciones, monitorear las cuentas para evitar la suplantación y los virus informáticos; la estrategia de evasión se basa en la negación a solicitar ayuda por miedo y cerrar las cuentas para cortar la comunicación con los acosadores, la estrategia de aceptación se caracteriza principalmente por la realización de actividades online que solicita el acosador y responder a todos los mensajes, la estrategia de enfrentamiento se fundamenta en el análisis de lo que le interesa al acosador, recopilar evidencias multimedia del ciberacoso, denunciar por escrito a las autoridades y bloqueo el contacto del acosador.

Partiendo de lo mencionado anteriormente, las estrategias utilizadas para frenar el ciberacoso deben además ser consideradas como responsabilidad del Estado, con campañas de sensibilización en torno a esta temática, donde los mensajes tanto en redes sociales como en medios de comunicación tradicionales se difundan sensibilizando a los usuarios sobre la temática, es decir como lo afirma (Carrera y Morejón, 2019) su labor es la de visibilizar la problemática. Entonces, las principales estrategias que usan los comunicadores sociales se enfocan en la prevención y llegan hasta el enfrentamiento para afrontar el acoso digital de acuerdo con el nivel del problema.

La investigación realizada se enfoca en las estrategias de comunicación desde la perspectiva de las víctimas, pero futuros estudios podrían contribuir con la identificación de las estrategias de los acosadores, espectadores y plataformas tecnológicas que vulneran la privacidad de los usuarios. 


\section{Referencias bibliográficas.}

Ballesteros, C. (2017). Los medios audiovisuales: funciones didácticas y principios metodológicos para su integración en los procesos de enseñanza y aprendizaje. Retrieved from https://rio.upo.es/xmlui/handle/10433/3507

Bégin, M. (2018). El Ciberacoso. Una revisión de investigaciones internacionales sobre representaciones, prevalencias, efectos y explicaciones del fenómeno. RePresentaciones. Investigación En Comunicación, (10), 52-78. Retrieved from http://revistas.usach.cl/ojs/index.php/re-presentaciones/article/view/3684

Bocij, P., Boci, P., \& Mcfarlane, L. (2002). Online harassment: Towards a definition of cyberstalking Online harassment: towards a definition of cyberstalkzng. Prison Service Journal, 31-38. Retrieved from www.harassmentlaw.co.uklbook!cyberep.htm.

Cañón, R., Grande, M., \& Ferrero, E. (2018). Ciberacoso: revisión de la literatura educativa en español Cyberbullying: literature review of educational literature in Spanish. Revista Latinoamericana de Tecnología Educativa, 17(2), 87-99. https://doi.org/10.17398/1695-288X.17.2.87

Carrera, M., \& Morejón, J. (2019). Comunicadoras y periodistas ecuatorianas: Alternativas para enfrentar el ciberacoso en el entorno digital | URU, Revista de Comunicación y Cultura. URU-Revista de Comunicación y Cultura, 2, 24-48. Retrieved from https://revistas.uasb.edu.ec/index.php/uru/article/view/1344/1215

Cerezo, F., \& Rubio, F. (2017). Medidas relativas al acoso escolar y ciberacoso en la normativa autonómica española. Un estudio comparativo. Revista Electrónica Interuniversitaria de Formación Del Profesorado, 20(1), 113. https://doi.org/10.6018/reifop/20.1.253391

Chiolero, A. (2020). Covid-19: A digital epidemic. The BMJ, 368. https://doi.org/10.1136/bmj.m764

Dinakar, K., Reichart, R., \& Lieberman, H. (2011). Modeling the Detection of Textual Cyberbullying. Proceedings of the International AAAI Conference on Web and Social Media, 5(1). Retrieved from www.aaai.org

Domínguez, C., Martínez-Pecino, R., \& Segura, M. (2015). Ciberacoso en la adolescencia y revelación de las agresiones. Apuntes de Psicología, 33(3), 95-102. Retrieved from http://www.apuntesdepsicologia.es/index.php/revista/article/view/567

Dueñas, D., Ponton, P., Belzunegui, Á., \& Pastor, I. (2016). Discriminatory expressions, the young and social networks: The effect of gender. Comunicar, 24(46), 67-75. https://doi.org/10.3916/C46-2016-07 
García, A., Tur-Viñes, V., \& Pastor, Y. (2018). Consumo mediático de adolescentes y jóvenes. Noticias, contenidos audiovisuales y medición de audiencias. Icono14, 16(1), 22-46. https://doi.org/10.7195/RI14.V16I1.1101

González, A. (2016). Factores de riesgo en el ciberacoso: revisión sistemática a partir del modelo del triple riesgo delictivo (TRD). IDP. Revista de Internet, Derecho y $\begin{array}{llll}\text { Política, } & 22, & 62-81 . & \text { Retrieved from }\end{array}$ https://www.redalyc.org/pdf/788/78846481006.pdf

Hernández-Sampieri, R., Fernández-Collado, C., \& Baptista-Lucio, P. (2010). Metodología de la investigación (Quinta). México D.F.: The McGraw-Hill.

Hernández Guarín, G., \& Castro Pacheco, A. (2014). Influencia de las redes sociales de internet en el rendimiento académico del área de informática en los estudiantes de los grados $8^{\circ}$ y $9^{\circ}$ del instituto promoción social del norte de Bucaramanga (Universidad del Tolima). Retrieved from https://core.ac.uk/download/pdf/51068081.pdf

Herrera, H. H. (2012). Las Redes sociales: Una nueva herramienta de difusión. Reflexiones, 91(2), 121-128. https://doi.org/10.15517/rr.v91i2.1513

Kitchenham, B. (2004). Procedures for Performing Systematic Reviews. Keele.

Obermaier, M., Hofbauer, M., \& Reinemann, C. (2018). Journalists as targets of hate speech. How German journalists perceive the consequences for themselves and how they cope with it. Studies in Communication | Media, 7(4), 499-524. https://doi.org/10.5771/2192-4007-2018-4-499

Organización Mundial de la Salud. (2020). Coronavirus Disease (COVID-19) Situation Reports. Retrieved June 11, 2021, from https://www.who.int/emergencies/diseases/novel-coronavirus-2019/situationreports

Peral-Peral, B., Arenas-Gaitán, J., \& Villarejo-Ramos, Á. (2015). De la brecha digital a la brecha psico-digital: Mayores y redes sociales. Comunicar, 23(45), 57-64. https://doi.org/10.3916/C45-2015-06

Rosa, H., Pereira, N., Ribeiro, R., Ferreira, P. C., Carvalho, J. P., Oliveira, S., ... Trancoso, I. (2019). Automatic cyberbullying detection: A systematic review. Computers in Human Behavior, 93, 333-345. https://doi.org/10.1016/j.chb.2018.12.021

Ruíz-Martín, A., Bono-Cabré, R., \& Magallón-Neri, E. (2019). Ciberacoso y ansiedad social en adolescentes: una revisión sistemática. Revista de Psicología Clínica Con Niños y Adolescentes, 6(1), 2019-2028. https://doi.org/10.21134/rpcna.2019.06.1.1

Tello, L. (2013). Intimidad y «extimidad» en las redes sociales. Las demarcaciones éticas de Facebook. Comunicar, 21(41), 205-213. https://doi.org/10.3916/C41-2013-20 
Torres, A., Manuel, J., \& de Marco, S. (2013). El ciberacoso como forma de ejercer la violencia de género en la Investigación promovida por la Delegación del Gobierno para la Violencia de Género. Madrid.

Tudela, S., \& Barón, A. (2017). Redes sociales: del ciberacoso a los grupos de apoyo online con víctimas de acoso escolar. Escritos de Psicología / Psychological Writings, 10(3), 167-177. https://doi.org/10.5231/psy.writ.2017.3011

Van Cleemput, K., K., Vandebosch, H., Poels, K., Bastiaensens, S., DeSmet, A., \& De Bourdeaudhuij, I. (2015). The development of a serious game on cyberbullying (Psychology; Cyberbullying: from theory to intervention, Ed.). Retrieved from https://books.google.com.ec/books?hl=en\&lr=\&id=m7_MCgAAQBAJ\&oi=fnd\&p $\mathrm{g}=\mathrm{PA} 93 \& \mathrm{dq}=\mathrm{Van}+$ Cleemput,$+\mathrm{K} .,+$ Vandebosch,$+\mathrm{H} .,+$ Poels,$+\mathrm{K} .,+$ Bastiaensens,$+\mathrm{S}$. ,+DeSmet,+A.,+\%26+De+Bourdeaudhuij, + I.+(2015).+The+development+a+seriou s+game+on+cyberbullying:+a+concept+test.+In+T

Van Royen, K., Poels, K., Vandebosch, H., \& Adam, P. (2017). "Thinking before posting?" Reducing cyber harassment on social networking sites through a reflective message. Computers in Human Behavior, 66, 345-352. https://doi.org/10.1016/j.chb.2016.09.040

Vanderhoven, E., Schellens, T., \& Valcke, M. (2014). Enseñar a los adolescentes los riesgos de las redes sociales: una propuesta de intervención en Secundaria. Comunicar, 22(43), 123-131. https://doi.org/10.3916/C43-2014-12

Verdejo, M. (2015). Ciberacoso y violencia de género en redes sociales: análisis y herramientas de prevención (U. I. de Andalucía, Ed.). Retrieved from https://dspace.unia.es/handle/10334/3528

Yang, F. (2021). Coping strategies, cyberbullying behaviors, and depression among Chinese netizens during the COVID-19 pandemic: a web-based nationwide survey. Journal of Affective Disorders, 281, 138-144. https://doi.org/10.1016/j.jad.2020.12.023

Young, R., Subramanian, R., Miles, S., Hinnant, A., \& Andsager, J. L. (2017). Social Representation of Cyberbullying and Adolescent Suicide: A Mixed-Method Analysis of News Stories. Health Communication, 32(9), 1082-1092. https://doi.org/10.1080/10410236.2016.1214214

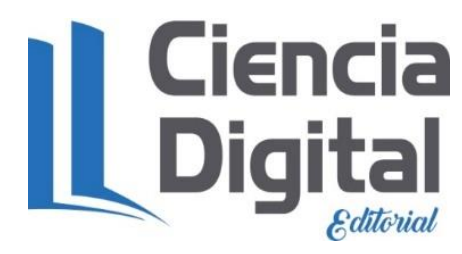




\section{PARA CITAR EL ARTÍCULO INDEXADO.}

Erazo Rodríguez, M. E., Viñan Carrasco, L. M., Murillo Naranjo, M. E., \& Jiménez Granizo , C. N. (2021). Estrategias de comunicación para enfrentar el ciberacoso en el contexto del COVID-19. ConcienciaDigital, 4(3.1), 23-40. https://doi.org/10.33262/concienciadigital.v4i3.1.1809

\section{Ciencia LDigital}

El artículo que se publica es de exclusiva responsabilidad de los autores y no necesariamente reflejan el pensamiento de la Revista Conciencia Digital.

El artículo queda en propiedad de la revista y, por tanto, su publicación parcial y/o total en otro medio tiene que ser autorizado por el director de la Revista Conciencia Digital.

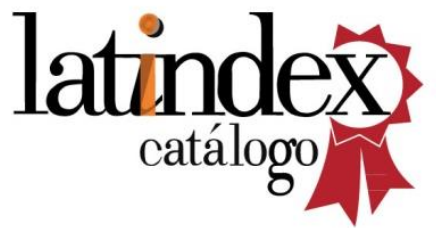

